\title{
Coherent laser radar for vibrometry: Robust design and adaptive signal processing
}

\author{
Ingmar Renhorn, Christer Karlsson, and Dietmar Letalick \\ National Defence Research Establishment (FOA) \\ Department of Sensor Technology \\ P.O. Box 1165, S-581 11 Linköping, Sweden \\ Tel: Int+4613 318000, Fax: Int+4613 331665 \\ Mille Millnert, Remke Rutgers \\ Linköping University \\ Dept. of Electrical Engineering \\ S-581 83 Linköping, Sweden \\ Tel: Int+4613 281681, Fax: Int+4613 149403 \\ e-mail: mille@isy.liu.se
}

\section{ABSTRACT}

A coherent laser radar system based on semiconductor laser technology has been designed and built. The compact design and the absence of adjustments makes the system mechanically robust and easy to use. The present system has an output power of $50 \mathrm{~mW}$ and a line width of $280 \mathrm{kHz}$ (HWHM). The laser radar system has been used in vibrometry measurements. For vibrometry of moving objects, adaptive signal processing is required in order to obtain the vibration signature. Especially for unresolved objects, interference between different vibrating parts will complicate the analysis. Modelbased estimation techniques are used to obtain the parameters which determine the dynamics of the reflecting object.

Key words: coherent laser radar, vibrometry, modelbased signal processing

\section{INTRODUCTION}

Coherent laser radar (CLR) systems have been investigated over several decades primarily for military applications. In order to receive a more general industrial acceptance, compact, robust, cost-effective systems and more general signal processing tools must be developed. Semiconductor laser technology can achieve both high power and narrow line width without using external cavity designs. This allows the design of very compact systems without any movable parts such as pzt-elements. A critical feature is the tolerance designing, allowing for laser welding of the interferometric block. The design is also compatible with distributed fiber sensor systems. The compact design and the absence of adjustments makes the system mechanically robust and easy to use. Semiconductor lasers can easily be broadband frequency modulated, and various applications can be addressed by making use of this property. For range/Doppler measurements, chirping or frequency shift keying can be applied. If necessary, a frequency shifted signal can, be obtained by using ,e.g., an acousto- optic modulator. CLR 
technologies are unique for remote sensing of rotational and vibrational characteristics of objects and surfaces. There are numerous and important applications in e.g. (jet)engine, helicopter, aircraft and car development and maintenance, wind tunnel instrumentations, power plant inspection and satellite vibrations. The vibrational signature can also be used for platform identification. The laser radar system measures the radial velocity of the surface at the focused spot. A vibrating surface will induce a small frequency (Doppler) shift of the reflected radiation. The Doppler shifted frequency is a measure of the instantaneous local velocity of the surface. The vibrational spectrum can be composed of several harmonic frequencies, induced by the vibration in different parts of the structure. This is the case for, e.g., helicopters, where the main rotor and tail rotor have different characteristics. Vibrometer systems are commercially available. Still, this is an area of extensive research. Most systems are based on heterodyne detection. Frequency shifts in heterodyne systems are obtained using basically three methods: 1) external frequency shifting in a Bragg cell, 2) separate local oscillator, 3) two-frequency laser. In homodyne systems, a frequency agile laser can be used, if necessary, to find the sign of the Doppler shift. Two limitations have been at the focus when developing the system presented here. First, to develop a small, simple and robust system with reasonable range capability against non-cooperative targets. Secondly, to develop signal processing techniques that will allow both limited control of the laser frequency and allow extraction of surface characteristics at low signal-to-noise ratios. Of the alternatives given above, the homodyne system is the simplest one to obtain a robust design, whereas using a separate local oscillator is the most complex one from this point of view. Homodyne detection results in an uncertainty of the sign of the Doppler shift. In many applications, however, the sign can either be obtained from other sensors or by frequency modulating the laser, or it is of no concern in the analyses. For moving targets, the constant Doppler shift has to be estimated. This shift can be used in resolving the sign of the vibration. A homodyne system can also, if necessary, be supplemented with a frequency shifting Bragg-cell. To obtain the vibrational signature various signal processing techniques can be used. For vibrometry of moving objects, adaptive signal processing is required in order to obtain the vibrational signature. Especially for unresolved objects, interference between different vibrating parts will complicate the analysis. In this paper, model based estimation techniques are used to obtain the parameters which determine the dynamics of the reflecting object.

\section{LASER SYSTEM DESIGN}

Coherent laser radar systems have been described in numerous papers. ${ }^{1}$ Cost effectiveness can be obtained by basing the system on $1.5 \mu \mathrm{m}$ laser technology. The critical parts of the system are the transmitter laser, the interferometric block, the local oscillator and the detector. As for the transmitter laser, line width and output power are the two most important parameters in a laser radar system. For monostatic systems, the beam expander and telescope will be less critical when it comes to inter- ferometric precision. In a homodyne system, the partial reflector will generate the necessary local oscillator power. In most designs, a flat partial reflector is placed in a collimated portion of the transmitter beam. This will result in very high angular precision requirements and very relaxed requirements on positioning along the optical axes. Here, in order to lower the requirement on angular tolerances, a balance has been sought between angular and position tolerances. It is possible to bring about such a trade off by focusing the transmitter beam on a partial reflector, choosing the focal length in such a way that tolerances adequate for micro-machining is obtained. This solution will allow the design of a compact coherent laser radar system at $1.55 \mu \mathrm{m}$ that is both robust and where adjustments will not be necessary. Most of the passive components have been developed for the communication industry and can be used without modification. The output radiation can also be amplified using an Erbium-doped fiber amplifier (EDFA). Modified single mode fibres for power amplification are available with high output power. The relatively short wavelength results in

high sensitivity for vibrations of small magnitude. A draw-back might be that the system will experience an increased sensitivity for atmospheric turbulence at very large ranges compared to e.g. $10 \mu \mathrm{m}$ systems. 


\section{EXPERIMENTAL DETAILS}

The measurements were performed using a laser radar based on a $1.55 \mu \mathrm{m}$ semiconductor laser. The present system has an output power of $50 \mathrm{~mW}$ and a linewidth of $280 \mathrm{kHz}$ (HWHM). A schematic diagram of this laser radar system, comprising the laser, the interferometric block and the detector, is shown in figure 1. A brief description of the system has been presented elsewhere. ${ }^{2}$

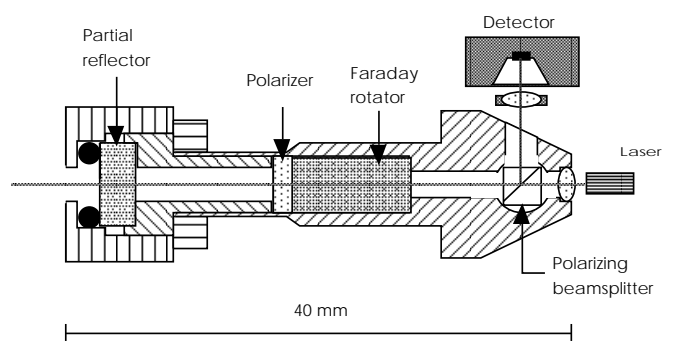

Figure 1: Basic structure of the semiconductor laser radar system.

The InGaAsP-InP laser is a three-section strained-layer quantum well distributed feedback (DFB) laser. ${ }^{4}$ The divergent beam from the laser is collimated by an aspheric lens such that minimal wave-front distortion is achieved. After passing the polarising beamsplitter cube and the Faraday element, which rotates the plane of polarisation $45^{\circ}$ , the major portion of the beam exits the radar. A small part (4\%) of the beam is reflected by the partial reflector. This reflex serves as the local oscillator (LO) necessary for coherent detection. Before mixing of the target and LO beams on the detector, both beams pass the Faraday element once more and are reflected by the beamsplitter cube. In this way, the important optical isolation of the laser is accomplished. The detector is a $25 \mathrm{~mm}$ diameter InGaAs Schottky photo diode. In order to be able to study interference phenomena, a target consisting of two identical bass loudspeakers, oriented mutually orthogonally, and a 50/50 non-polarising beamsplitter cube was constructed (Fig. 2). Small pieces of ordinary copy paper, sprayed with aluminum paint, were affixed to the membranes of the loudspeakers. The $\mathrm{Al}$ painted paper have similar reflectance properties as sandblasted aluminum and thus the loudspeakers act as vibrating diffuse surfaces. The loudspeakers were characterized, with respect to overtones, for the amplitudes and frequencies of the drive signals using a microphone. In all cases, the overtones were substantially lower than the fundamental harmonic. Also the measurements with the laser radar indicated that the amplitudes of the overtones were negligible.

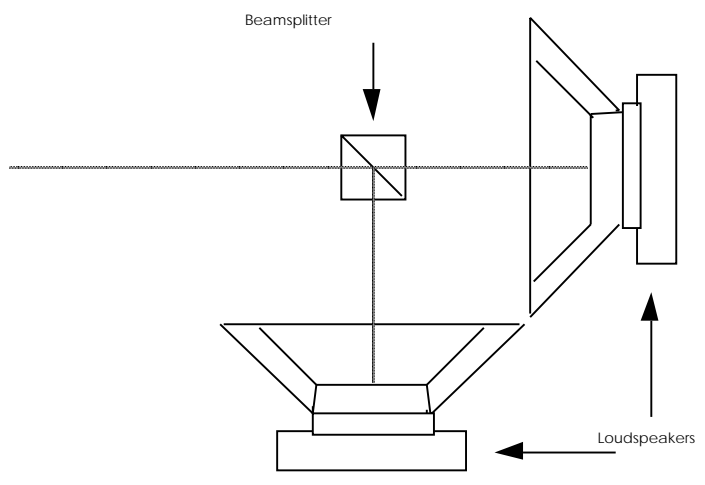

Figure 2: Target used to study vibrational interference effects.

The location of the target was 2 meters from the laser radar. In order to increase the homodyne signals, a lens $(\mathrm{f}=160 \mathrm{~mm})$ was used to focus the collimated beam onto the membranes. The laser was operated such that the line width and power were $\approx 1 \mathrm{MHz}$ and $10 \mathrm{~mW}$, respectively. All wave forms presented in this paper are 
obtained under these conditions. The wave forms were recorded with a 8-bit digital oscilloscope. For each set of test parameters (signal amplitudes and frequencies for the loudspeakers), the resulting homodyne signal for each loudspeaker was recorded separately, by obstructing the path to the other loudspeaker, along with the total homodyne signal (both loudspeakers unobstructed). It is important to actually measure the homodyne signal for each loudspeaker since the Al-sprayed paper gives rise to a speckle pattern which in turn results in homodyne signal amplitudes which vary depending on the exact positioning of the laser beam on the target. Thus, by the course of action described above, a comparison can be made between the results of the signal analysis of the complex total homodyne signal with the correct signals from each loudspeaker. The shifted and filtered signal is digitized in order to allow for flexible signal processing. In practice, many samples must be taken over the highest period to be analyzed. Oversampling is necessary due to the speckle noise that will be present in all measurements on diffuse targets. In low $\mathrm{S} / \mathrm{N}$ measurements, there will not be possible to determine the signal phase. Instead, the instantaneous frequency is determined over a limited time window. The time window is optimized with respect to the characteristics of the expected signal. A block diagram of the processing scheme is given in Fig 3.

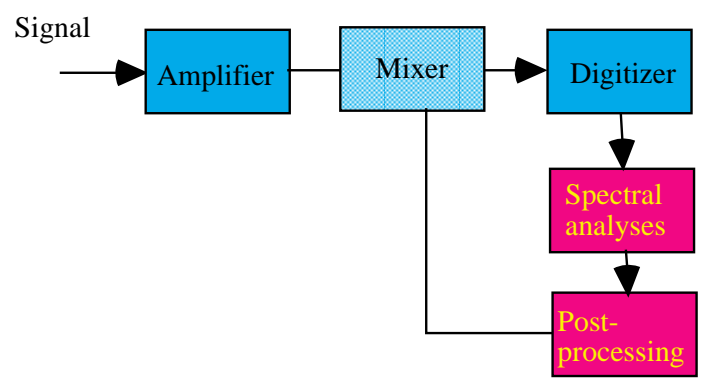

Figure 3: Signal processing block diagram.

\section{HOMODYNE DETECTION}

The homodyne technique is adopted in order to obtain a very simple and rugged system. In many applications this will be an adequate technology. The homodyne signal is centered around the zero frequency. Many systems exhibit substantial $1 / f$-noise in this region. At relatively short wavelength, good signal-to-noise ratios can be obtained also in this region. Even when the lowest frequencies are filtered out with a high pass filter, the signal will exhibit only moderate distortion in an uncritical frequency region.

A vibrating surface will generate a detectable Doppler shift. The instantaneous frequency is determined by the vibrational frequency and vibrational amplitude of the surface. If the position of the surface is given by $x(t)$, a harmonic description apart from a phase factor is

$$
x(t)=x_{0} \sin \left(\omega_{v} t\right)
$$

where $\omega_{v}=2 \pi f_{v}, f_{v}$ is the vibrational frequency and $x_{0}$ is the vibrational amplitude. The instantaneous Doppler shift is given by

$$
\begin{aligned}
v_{v}(t) & =\frac{2}{\lambda} \frac{d x(t)}{d t} \\
v_{v}(t) & =v_{D}(t) \cos \left(2 \pi f_{v} t\right) \\
v_{D}(t) & =\frac{4 \pi x_{0} f_{v}}{\lambda}
\end{aligned}
$$

For a moving target, a constant frequency shift will be superimposed on the vibrationally induced Doppler 
shift. For such targets, there is basically no difference from a signal processing point of view for a hetero-dyne system and a homodyne system. The traditional signal processing method is to use an FM-detector that is optimized for the relevant signal. This method works well at large signal-to-noise ratios $(\mathrm{S} / \mathrm{N}>10 \mathrm{~dB})$. In many applications, the $\mathrm{S} / \mathrm{N}$ will be low, at least occasionally, due to speckles. A more robust signal processing technique that will allow also low $\mathrm{S} / \mathrm{N}$ ratios is therefore desirable.

The instantaneous frequency is given by the Doppler shift from the radial velocity of the target and the (usually much smaller) Doppler shift induced by the vibrating surface. The signal can, for a harmonic vibration, be described by

$$
s(t) \approx C \cos \left(2 \pi v_{c} t+\mu \sin \left(2 \pi f_{v} t+\theta_{v}\right)+\theta\right)
$$

where $\mu$ is the modulation index and the vibration is periodic with the frequency $f_{n}$. Most of the signal power will be within the bandwidth

$$
B \approx 2(\mu+1) f_{v}
$$

The signal $s(t)$ can be shifted by suitable mixing with an oscillator to a frequency region that is convenient for signal processing. What is meant by suitable depends on the application. This process is described below.

\section{PARAMETER ESTIMATION}

In this section the method for estimating the interesting parameters in the signal model will be given. In order to evaluate the estimation procedure it will be applied to simulated data. For more details see. ${ }^{5}$ A signal model consisting of signal components is given by

$$
y(t)=\sum_{n=1}^{n_{s}} s_{n}(t)+e(t)
$$

The signal components are given by

$$
s_{n}(t)=C_{n} \cos \left(\omega_{c}^{(n)} t+\mu_{n} \sin \left(\omega_{v}^{(n)} t+\theta_{v}^{(n)}\right)+\theta_{c}^{(n)}\right.
$$

where the parameters $\left(\begin{array}{llll}C_{n} & \omega_{c}^{(n)} & \mu_{n} & \theta_{v}^{(n)}\end{array}\right)$ for the signal components are to be estimated. In figure 4 the two signal components of the simulated data used for illustration are shown. In figure 5 the signal obtained as the sum of the two signal components is shown.

The first step in the estimation procedure is to calculate the short time windowed Fourier transform and the corresponding power spectra, i.e., the spectrogram of the signal, see figure 6 below.

The fundamental problem of the parameter estimation is to separate the two signal components. The modulation frequency of each signal component gives a sinusoidal "ridge" in the spectrogram of the form

$$
\omega_{v}^{(n)} \mu_{n} \sin \left(\omega_{v}^{(n)} t+\theta_{v}^{(n)}\right.
$$

i.e., a sinusiod with frequency $\omega_{v}^{(n)}$ and amplitude $\omega_{v}^{(n)} \mu_{n}$. Component separation is achieved by calculating the mean frequency of the spectra for each time slot in the spectrogram, i.e.,

$$
\bar{\omega}(t)=\frac{\sum_{\omega>0}|Y(\omega, t)|^{2} \omega}{\sum_{\omega>0}|Y(\omega, t)|^{2}}
$$

In figure 7 the resulting signal is shown. The next step is to calculate the Fourier transform of (10) which will clearly display the frequencies of the two signal components as peaks, see figure 9 , The vibration frequencies and phase-shifts can now be extracted using standard techniques, see. ${ }^{5}$ 

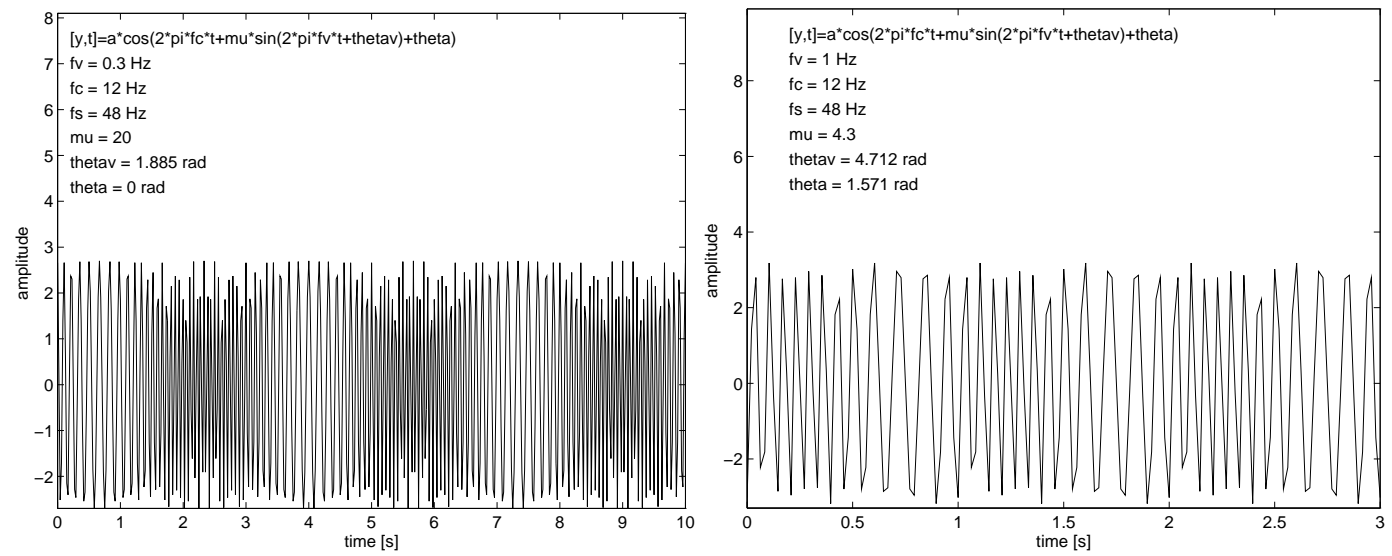

Figure 4: The figure shows the simulated signal components used for illustrating the parameter estimation procedure.

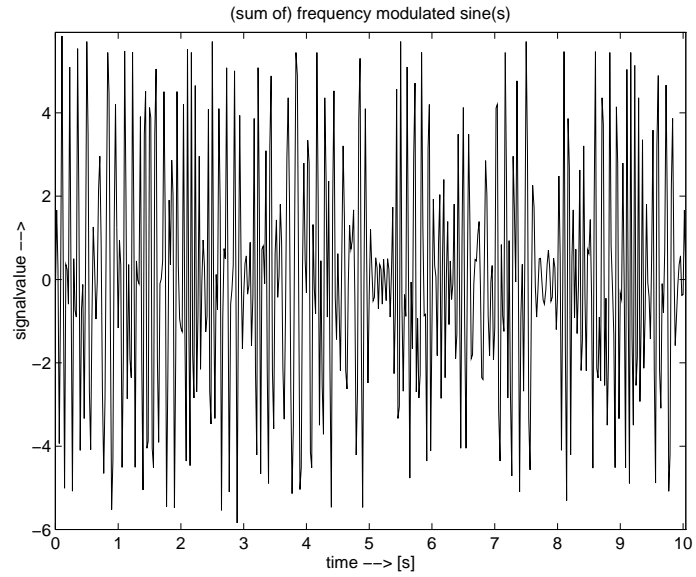

Figure 5: The signal obtained by adding the signal components shown in figure 4

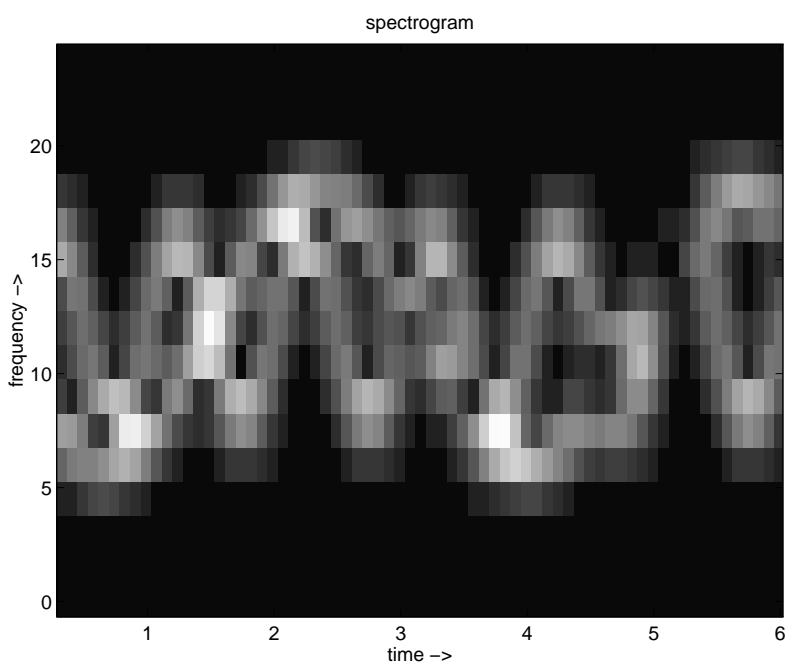

Figure 6: The spectrogram of the signal in figure 6 based on a 32-sample FFT using a Hamming window. 


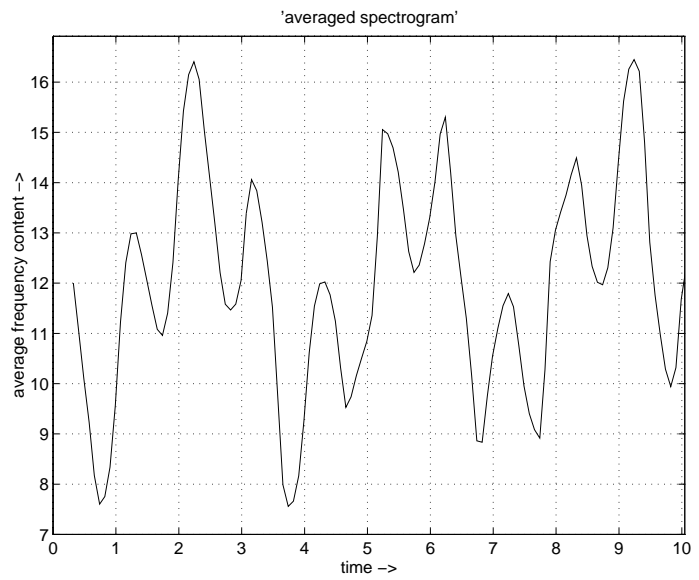

Figure 7: The mean frequency of the spectrogram in figure 6 .
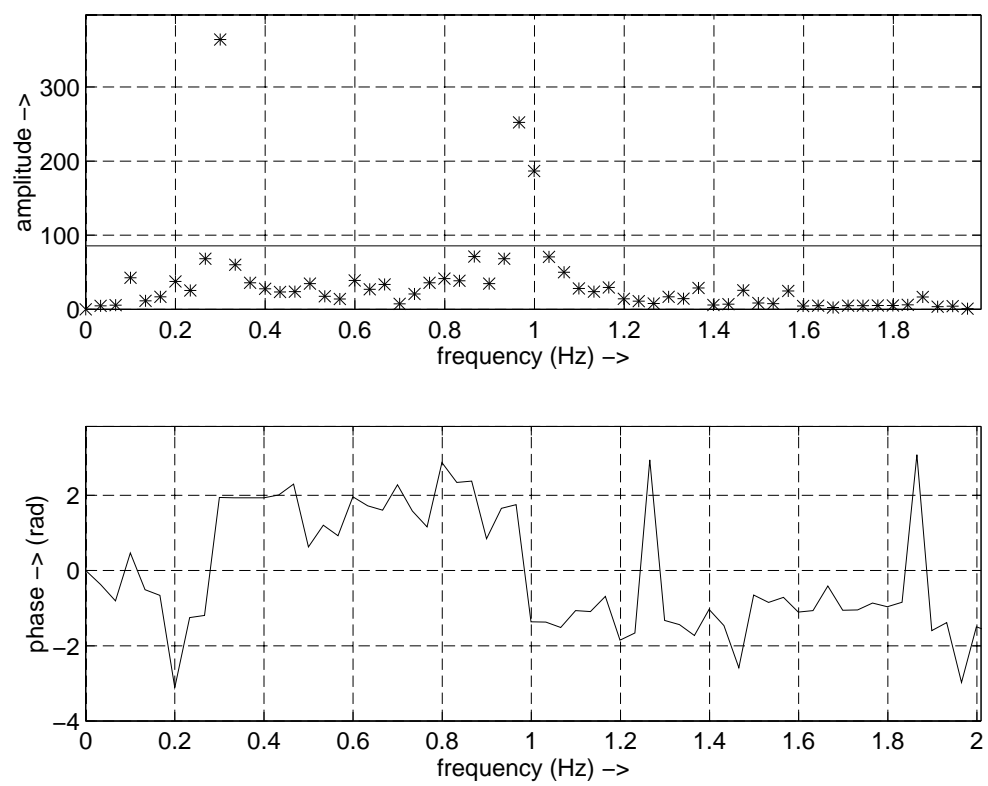

Figure 8: The Fourier transform of the signal in figure 7 
By fitting the models (7) to the spectrogram in such a way that the accumulated energy of the corresponding time-frequency bins is maximized the quantities

$$
\omega_{v}^{(n)} \mu_{n}
$$

can be estimated. Since the modulation frequencies already are known this gives the modulation indices. Moreover, from the so far obtained estimates and the total signal mean energy the amplitude of the signal components can be estimated, see. ${ }^{5}$ In table 1 the correct and estimated values of the signal parameters. For more information $\operatorname{see}^{5}$

\begin{tabular}{|c|c|c|c|c|c|}
\hline \multirow{2}{*}{ signal 1} & & Mod. freq. $\omega_{v}^{(n)}$ & Mod. index $\mu_{n}$ & Mod. phase $\theta_{v}^{(n)}$ & Amplitude $C_{n}$ \\
\hline & true values & 0.3 & 20 & 1.885 & $\begin{array}{c}2.7 \\
245\end{array}$ \\
\hline signal 2 & true values & 1 & 4.3 & 4.712 & 3.3 \\
\hline & estimated values & 1.001 & 3.00 & 4.653 & 3.22 \\
\hline
\end{tabular}

Table 1: The correct and estimated values of the parameters in the signal model.

\section{$7 \quad$ CONCLUSIONS}

A homodyne laser radar system has been used for detecting surface vibrational characteristics at low signal-tonoise ratios. It was possible to overcome the limitations of conventional FM-detection by using spectral analysis techniques. This is especially beneficial when the signal is contaminated by interfering signals and speckle noise. If the target is translating or rotating, speckle noise will be introduced into the demodulated signal . Speckles cause large fluctuations in both amplitude and phase. Substantial degradation is experienced when the frequency broadening due to speckle noise is of the same order of magnitude as the vibrationally induced signal bandwidth.

Practical systems utilizing lasers with relatively short wavelength, such as the system presented here, will also exhibit larger speckle noise than systems using longer wavelengths. These circumstances arise from the longer wavelength that will give rise to a larger spot size at the target due to diffraction and that the effective aperture is larger at longer wavelengths due to turbulence limitations. On the other hand, the vibrationally induced bandwidth will be larger at shorter wavelength. There will therefore be a trade-off between range requirement and wavelength. Speckle noise will show up at both low translational speeds $(<5 \mathrm{~m} / \mathrm{s})$ and low rotational speeds $(<0.015 \mathrm{rad} / \mathrm{s})$. The speckle noise makes the conventional FM receiver less attractive since it gives rise to the phenomena known "click noise".

\section{REFERENCES}

[1] R. G. Frehlich and M. J. Kavaya, "Coherent laser radar performance for general atmospheric refractive turbulence." Appl. Opt., Vol. 36, 5325-5352. (1991)

[2] D. Letalick, I. Renhorn, O. Steinvall, and J. Shapiro, Noise sources in laser radar systems, Appl. Opt., Vol. 28, 2657-2665 (1989).

[3] I. Renhorn, B. Broberg, and K. Vilhelmsson, Coherent laser radar based on semiconductor laser technology, CLEO Europe 1994, Amsterdam.

[4] U. Öhlander, D. Karlsson Varga, S. Nilsson, J. André, J. Wallin, S. Lindgren, S. Lourdudoss, O. Kjebon, B. Broberg and G. Landgren, Three-section GRIN-SCH SL-MQW $1.55 \mathrm{~mm}$ SI-DFB lasers for coherent telecommunications, ECOC 1992, Berlin. 
[5] R. Rutgers, "Parameter estimation in vibrometry data". Report: LiTH-ISY-R-1745. (1995) 